\title{
RESPONSABILIDAD SOCIAL UNIVERSITARIA: UNA MIRADA DESDE AMÉRICA LATINA Y COLOMBIA ${ }^{1}$
}

\author{
Maira Alejandra Sissa Dueñas ${ }^{2}$
}

Para citar este artículo: Sissa, M. (2015). Responsabilidad social universitaria: una mirada desde América Latina y Colombia. Inquietud Empresarial. Vol. XV (2), 65-85.

Fecha de recepción: 2 de junio de 2015 Fecha de aceptación: 13 de noviembre de 2015

\footnotetext{
Artículo de revisión como resultado del proyecto de investigación titulado "Aproximación a un estado del arte de responsabilidad social universitaria en América Latina y principales avances de algunas universidades en Colombia". En desarrollo con el semillero de investigación IDEAS de la Facultad de Ciencias Económicas y Administrativas. Universidad Pedagógica y Tecnológica de Colombia.

2 Administrador de empresas, Universidad Pedagógica y Tecnológica de Colombia, Integrante del grupo de investigación Ideas, mairasissa27@gmail.com
} 


\title{
Resumen
}

La responsabilidad social ha estado presente a nivel nacional e internacional desde hace algunas décadas, por ello, que en este trabajo, en primer lugar se da un acercamiento teórico de lo que encierra la responsabilidad social, desde su conceptualización hasta las normas e instituciones que la regulan. Por su parte, las universidades han querido sembrar esta responsabilidad social, por lo que posterior a este recuento de responsabilidad social, se da a conocer la Responsabilidad Social Universitaria RSU. Por medio del objetivo principal de esta investigación (contribución al análisis de la responsabilidad social universitaria en América Latina), se muestran los puntos de vista de algunos autores que han indagado y hecho investigaciones alrededor de este tema. Existen un sin número de universidades en América Latina y Colombia, con la que se hace una investigación de que han hecho, para apropiarse y buscar la RSU dentro de sus universidades, estas proyectan su imagen ante la sociedad, pero es solo sus impactos las que las definen, de forma negativa o positiva por consiguiente es importante que las universidades guíen su actuar éticamente con valores y principios.

Palabras clave: responsabilidad social, responsabilidad social universitaria, proyección social, extensión universitaria, misión universitaria, ética, impactos.

\section{Social University responsibility: a look from Latin America and Colombia}

\begin{abstract}
Social responsibility, has been presented at the national and international level for some decades. That is why, in this work, on the first hand, a theoretical approach is given to what social responsibility encompasses, from its conceptualization to the rules and institutions that regulate it. On the other hand, universities have wanted to sow this social responsibility and after this social responsibility account, University Social Responsibility RSU is announced. By means of the main objective of this research (contribution to the analysis of university social responsibility in Latin America), the points of view of some authors who have investigated and made research on this subject are shown. There are some universities in Latin America and Colombia, with which an investigation is made of what they have done, in order to appropriate
\end{abstract}


and seek the RSU within their universities. They project their image towards society, but it is only their impacts that define negatively or positively. For this reason, it is important that universities guide their action ethically with values and principles.

Key words: social responsibility, social responsibility university, social projection, university extension, university mission, ethics, impacts.

\section{Responsabilidade social universitária: uma visão da América Latina e da Colômbia}

\section{Resumo}

A responsabilidade social tem estado presente em nível nacional e internacional durante várias décadas, o que levou à necessidade de se apresentar inicialmente uma abordagem teórica sobre o tema responsabilidade social, desde sua conceituação até às normas e instituições que a regula. Por outro lado, as universidades buscaram semear esta responsabilidade social, uma vez que foi divulgada a Responsabilidade Social Universitária RSU posterior a esse trabalho. Através do objetivo principal desta pesquisa (contribuição para a análise da responsabilidade social universitária na América Latina), são apresentados os pontos de vista de alguns autores que têm questionado e realizado pesquisa em torno deste tema. Há diversas universidades na América Latina e na Colômbia que vêm realizando pesquisas como esta para se apropriar e buscar a RSU dentro de suas universidades, as quais projetam sua imagem frente à sociedade, mas são apenas os seus impactos que as definem, de forma negativa ou positiva, tornando importante que as universidades guiem sua atuação eticamente com valores e princípios.

Palavras chave: responsabilidade social, responsabilidade social universitária, projeção social, extensão universitária, missão universitária, ética, impactos. 


\section{Introducción}

Vivimos en una sociedad que es hostigada por todo tipo de información, para el caso de la responsabilidad social se puede encontrar infinidad de construcciones teóricas que se relacionan al tema, aunque es difícil dar un único concepto, donde puede ocurrir que se llegue a la confusión para quien esté interesado en indagar más a fondo. El propósito principal es, el de acercarnos a comprender el contenido central de esta investigación que es la responsabilidad social de las universidades (RSU), que en primera instancia se analiza de manera muy generalizada las diferentes reflexiones de autores y organismos internacionales que han hecho que la responsabilidad surja y tome fuerza a lo largo de esta última década, presentando de forma práctica y contextualizada una recopilación teórica de la responsabilidad social.

Por un lado, en cuanto a las responsabilidad social, es importante iniciar señalando un concepto, este por medio de Vallaeys (2013) quien la define como "una nueva política de gestión, un nuevo modo de administrar las organizaciones, cuáles sean, cuidando de los impactos y efectos colaterales que se generan a diario adentro y afuera de la institución, responsabilizándose por las consecuencias sociales inducidas por el mismo funcionamiento de la organización" las instituciones independientemente de su accionar, deben estar atentas a las consecuencias que se generan de su actividad tanto dentro como fuera de ellas, estas deben ser responsables en el desarrollo de políticas para gestionar los efectos a los que puede conllevar el funcionamiento en el ejercicio de la acción organizacional. Por su parte, en términos más sencillos el reconocido Drucker (2006), expresa que las organizaciones asumen una responsabilidad con sus empleados, el medio ambiente, los clientes y en general los que se vean implicados con la organización, caracterizando los actores del proceso de responsabilidad social.

Estas personas o grupos implicados pueden verse afectadas por las acciones de la empresa, lo que se hace referencia es a los stakeholders -grupos implicados con la empresa- Martínez (2011), donde Richard Edward Friedman citado en González (2007) define como "cualquier grupo o individuo que puede afectar o ser afectado por el logro de los objetivos de la empresa”, en donde se destaca que las empresas ven la necesidad de tomar conciencia entendida esta como una co-responsabilidad.

De manera crítica, por su parte Milton Friedman sostiene que el rendimiento económico es la única responsabilidad de un negocio, esto con el argumento que al no existir responsabilidad se desperdician los recursos de la misma sociedad (Drucker, 1995). 
Por lo que es posible reconocer, que los diferentes puntos de vista, muestran que con esta responsabilidad se busca que en especial las organizaciones den un primer paso hacia desarrollo de la sociedad, por una parte en cuanto a su manera de actuar en su entorno y por la otra a su sostenibilidad que guíe el desarrollo económico del país, como lo expresa De la Red Vega (2009) al decir que es cuestión de que se incluyan procesos y resultados, por medio de actitudes y acciones de cada uno de los miembros de la organización.

Cabe aclarar que en el tema de responsabilidad social se tiende a la confusión en cuanto a Responsabilidad Social Empresarial RSE, se identifica por los deberes éticos que tiene la empresa, relacionada con la normatividad que se da a través de los actores en el ejercicio de la actividad organizacional, por lo cual se derivan impactos generales de los que las empresas son responsables y la Responsabilidad Social Corporativa RSC, inicia por el cumplimiento de las normas nacionales e internacionales actuales, a partir de ellas medir y cuantificar los impactos, el compromiso ético con las demás empresas y los grupos con los que se relaciona (Castro, 2005).

\section{Normas e iniciativas internacionales de responsabilidad social}

Es preciso mirar algunas normas e iniciativas internacionales de responsabilidad social a manera de información:

La Comunidad europea de Naciones (2001) en su libro verde, crea en Europa con el objetivo de comenzar un debate sobre cómo la Unión Europea que fomentará la responsabilidad social de las empresas en Europa y a nivel internacional, en particular sobre "cómo aprovechar al máximo las experiencias existentes, fomentar el desarrollo de prácticas innovadoras, aumentar la transparencia e incrementar la fiabilidad de la evaluación y la validación”.

a. The Organisation for Economic Co-operation and Development (OECD): se fundó en 1991, cuya traducción es "Organización para la Cooperación y el Desarrollo Económicos (OCDE)", ellos realizan un foro en el que gobiernos comparten experiencias, trabajan en conjunto para dar soluciones a sus problemas comunes. La misión es la promoción de políticas que mejoren el bienestar social y económico de las personas en el mundo (Organización para la Cooperación y el Desarrollo Económicos, 2014).

b. Social Accountability International - SAI, (2015): organización sin ánimo de lucro, surge hacia el año 1997 y brinda formación por medio de capacitación en 
responsabilidad social, también ayuda a que no se violen los derechos de los trabajadores en el mundo ya que busca proteger la integridad de los mismos, esta organización trabaja mutuamente con empresas, sindicatos, y organizaciones no gubernamentales. Con la certificación SA 8000 siendo esta una de sus primeras normas de certificación social, basada en la OIT, la Organización de las Naciones Unidas ONU y los derechos humanos, donde se crea un lenguaje común para medir el desempeño social.

\section{Metodología}

Para el desarrollo de este escrito, se tuvieron en cuenta publicaciones realizadas a partir del 2009 hasta el 2014, autores que han hablado de responsabilidad social universitaria en América Latina y con limitación en la herramienta de búsqueda a Scopus, Ebsco Host (Business Source Premier, e- libro, Fuente Académica), ProQuest y Redalyc. Para un mejor análisis, se revisó lo que están haciendo las universidades latinoamericanas frente a la RSU, para ello se tomaron como referencia las 20 mejores universidades de América Latina ${ }^{3}$

Siguiendo con la investigación, para hacerle un análisis a Colombia, se tuvo en cuenta los datos encontrados en Sapiens Research ${ }^{4}$, del Rankin publicado el 4 de septiembre de 2014, adicional a esto, las universidades seleccionadas contaron con el criterio que fueran universidades acreditadas de alta calidad para el año 2014 (datos encontrado en $\mathrm{CNA}^{5}$ ). Para su análisis, se tomó una de las cuatro áreas propias de la RSU (docencia, investigación, extensión y gestión), en este caso se tomó extensión o proyección social.

Se realizó el análisis de extensión universitaria en las principales universidades de Colombia; el tema de RSU busca ir más allá, se tuvo en cuenta que para el análisis de lo que se ha avanzado, se tomaron publicaciones hechas en revistas indexadas en Scopus y Publindex que estén en categoría A1 y A2.

$3 \quad$ Dato que ofrece el QS TOPUNIVERSITIES Worldwide university ranking: se destacan las mejores universidades de América Latina en el 2014 teniendo en cuenta indicadores de investigación.

4 Publica cada semestre el Ranking U-Sapiens, clasificación de mejores universidades de Colombia, este muestra la clasificación de universidades colombianas según indicadores de investigación, donde se consideran criterios de las publicaciones académicas hechas en revistas indexadas en Publindex, su oferta de maestrías y doctorados, y los grupos de investigación con los que cuentan.

5 El Consejo Nacional de Acreditación (2014), organismo de naturaleza académica que depende del Consejo Nacional de Educación Superior (CESU), integrado por personas de las más altas calidades científicas y profesionales, cuya función esencial es la de promover y ejecutar la política de acreditación adoptada por el CESU y coordinar los respectivos procesos. 


\section{Resultados}

\subsection{Un acercamiento a la contextualización de la RSU vista desde publicaciones Latinoamericanas}

Haciendo un recuento en la historia, los aportes que más sobresalen parten del año 1990 en donde se firmó la Declaración de Talloires (2005), menciona que las universidades deben ser líderes y apoyar a la sociedad por medio de una buena educación, investigación y formación política, pero es asunto de cada universidad como responda socialmente con sus estudiantes, maestros, egresados, investigadores y ciudadanos. En la declaración de Talloires, hace énfasis en que las universidades deben promover los valores humanos a toda su comunidad universitaria para que sean compartidos localmente, es decir, a la sociedad a la cual pertenecen nacionalmente y que trasciendan globalmente, todo esto respaldado claro está por estas instituciones, logrando así que se fortalezca la democracia y la justicia (apoyando a marginados y reduciendo la pobreza).

Otro aporte es dado por la Organización de las Naciones Unidas para la Educación, la Ciencia y la Cultura (1998), plantea en la Declaración Mundial sobre la Educación Superior para el siglo XXI: "la educación superior debe reforzar su servicio a la sociedad y en especial sus actividades para eliminar la pobreza, la intolerancia, la violencia, el analfabetismo, el hambre, la degradación ambiental y la enfermedad, utilizando principalmente enfoques transdisciplinarios e interdisciplinarios en el análisis de los temas y los problemas".

El aumento de universidades en esta década ha sido significativo, pero más que en cifras lo que cabe resaltar, es que cada vez muchas de estas universidades empiezan a tomar conciencia de lo que sus acciones y comportamientos diarios generan en la sociedad, a esto es lo que se le ha venido dando la denominación de Responsabilidad Social Universitaria, autores como Casilla y Camacho (2012) plantean como un compromiso que la universidad hace para desarrollar proyectos orientados a la solución de problemas, crear lazos con organizaciones públicas, privadas y en general con la comunidad, logrando así la sostenibilidad.

Por su parte Herrera, Didriksson y Sánchez (2009) ven la RSU como "la relación entre el papel desempeñado por las instituciones de educación superior para formar recursos humanos y generar conocimiento, y lo que la sociedad demanda”, esto con proyectos de bienestar y desarrollo humano sostenible, respondiendo a necesidades que hay en el entorno de manera oportuna, eficaz y ética. Cavero (2006) citado en Vallejo y Govea (2011) manifiesta que "es un enfoque ético del vínculo mutuo entre 
universidad y sociedad", lo que busca es crear en las universidades un compromiso moral donde se generen nuevos conocimientos que ayuden a dar solución a problemas y exista humanismo.

Adicional a esto como lo expresa Aguirre, de Pelekais y Paz (2012) internamente la comunidad universitaria debe proyectar sus valores sociales, pero primero siendo responsable dentro de la misma universidad, lo que implica que la universidad debe comportarse bajo un estándar ético y moral, más que algo voluntario, se propone que la RSU sea un indicador de gestión en las instituciones. En cuanto al medio externo, la universidad como lo mencionan Bernal y Rivera (2011) es parte de una sociedad que cumple objetivos concretos y que realiza funciones en relación con esta, dichas funciones de la universidad como lo manifiesta, García y Alvarado (2012), Castañeda, Ruiz, Viloria, Castañeda y Quevedo (2007), son la docencia, la investigación, la gestión y la extensión, que cumplen con "un compromiso institucional con la comunidad" respaldado por comportamientos éticos y valores, entonces con las apreciaciones de estos autores se precisa que:

a. Por medio de la Docencia se pueden realizar proyectos de desarrollo, produciendo nuevos conocimientos y sirviendo a la población con acciones que mejoren su calidad de vida. Martínez y Hernández (2013), manifiestan que el docente debe tener la capacidad de enseñar por competencias y valores, creando habilidades a quienes está educando, Vera, Gómez, Acosta y Perozo, (2012), opinan que los profesores son los principales autores y ellos reflejan dedicación completa, donde ellos mismos son capaces de generar productos tangibles de pertinencia social, a esto ellos le dan una denominación de función científica.

b. Por otro lado está la Investigación donde actores externos, estudiantes y maestros fomenten el desarrollo sostenible y resolución de problemas por medio de la producción y difusión de conocimientos. Vallejo y Govea (2011) opinan que para exista una buena investigación esta debe tener como primera instancia logros que propicien la interacción con la sociedad contribuyendo al desarrollo sustentable y una cultura de paz, también debe tener apoyo, asesoramiento y servicio a la comunidad, como tercer criterio una participación de los diferentes actores sociales con los que se puede hacer un mejor desarrollo académico, en cuarto lugar plantea la investigación como una actividad productiva que trasciende desde universidades hasta países cumpliendo el fin de responsabilidad social.

c. La Gestión, está dada a la manera que la universidad da un buen manejo de sus recursos, sus procesos administrativos, sus políticas ambientales y laborales, en donde estas sean ejemplo para toda la comunidad cuando se habla de extensión universitaria. 
d. Por último, esta la Extensión o Proyección Social donde se busca generar alianzas estratégicas con la comunidad por medio de asesoría y servicio, es una especie de práctica con la realidad. (Herrera y otros 2009) destacan algunos propósitos, donde se encuentran por mencionar algunos:

- Relacionar a la universidad con la sociedad mediante la difusión y extensión del humanismo, la ciencia, la tecnología y otras manifestaciones de la cultura.

- Promover en los universitarios el fortalecimiento y perfeccionamiento de una conciencia de responsabilidad y compromiso con la sociedad, así como de una identidad con la universidad.

- Constituir, preservar, incrementar, administrar y divulgar el patrimonio cultural universitario y el acervo humanístico, científico y tecnológico.

- Identificar e investigar los problemas y demandas de la sociedad, para mejorar su calidad de vida.

Por otro lado, hay opiniones de críticas, pero de igual importancia, por ejemplo al decir que universidad está en una zona de confort en cuanto a responsabilidad social se refiere, esto en términos de Vallaeys (2014), quien manifiesta que para ciertas organizaciones es indispensable la responsabilidad social, caso que para las universidades no, es más un "compromiso ético" por parte de la universidad, por que como lo manifiesta el autor la universidad es la creación de "meta-conocimientos", es decir, "conocimientos que garantizan que los conocimientos sean tales, y no opiniones, ocurrencias o fantasías".

Por su parte Gasca y Olvera (2011) manifiestan que lastimosamente hoy en día la universidad es vista como un ente que vende formación, donde se compite por un empleo, ganar dinero y donde se convierte al universitario en un consumidor más, lo que supone interrogantes de como desde las aulas se está tratando el tema de responsabilidad social. Siguiendo con esos puntos de vista González, Pirela y Zerpa (2012) por su parte ven que el sistema educativo posee fallas ya que analizan que los países latinoamericanos presentan grandes cambios sociales, económicos que les exigen innovación, dicho sistema educativo busca generar buena convivencia con la sociedad en general, esta falta se observa también en cuanto se pasa por alto que la que la formación docente es de gran importancia y no se le presta mayor atención; estos autores también hablan de unos principios que guían la responsabilidad social para el cambio educativo en América Latina, donde el estudiante o docente tome conciencia de no ser un "consumidor de conocimiento", sino un generador.

Recogiendo todos estas apreciaciones se pude concluir que la RSU integra cuatro grandes pilares, la investigación, gestión, extensión y docencia, también involucra a 
muchos actores entre ellos los estudiantes, docentes, administrativos y otro personal afectado, y esta complementa la misión de educación de las Instituciones de Educación Superior, pero aún no se ha dicho la última palabra ya que la RSU va más allá, como se verá a continuación:

\subsection{Entonces... ¿qué más abarca la RSU?}

Al referirse a esta pregunta, se pone en manifiesto que la RSU va más allá de su parte conceptual, de este modo se observa que existe una correlación de la RSU, con cada elemento que la compone es importante ya que cada uno intenta responder a una situación que enfrenta la universidad con la sociedad a la que pertenece, para que se realicen estrategias propias de mejoramiento como lo menciona Vallaeys (2013). Se muestran de manera detallada las áreas que están en sinergia con la RSU.

Tabla 1. Áreas que abarca la RSU

\begin{tabular}{|l|l|}
\hline \multicolumn{1}{|c|}{ Área } & \multicolumn{1}{c|}{ CARACTERÍSTICA } \\
\hline Ética & $\begin{array}{l}\text { Involucra valores éticos que se relacionan con el actuar de la } \\
\text { universidad cumpliendo una función social. }\end{array}$ \\
\hline Crecimiento Económico & $\begin{array}{l}\text { Formación de profesionales innovadores, activos, que } \\
\text { demanda la sociedad. }\end{array}$ \\
\hline Medio Ambiente & $\begin{array}{l}\text { Entorno natural, social, y cultural donde la universidad } \\
\text { desarrolla sus actividades. }\end{array}$ \\
\hline Equidad Social & $\begin{array}{l}\text { Por medio de un modelo equitativo distribuir capacidades, } \\
\text { igualdad de acceso, permanencia y desempeño estudiantil y } \\
\text { acceso universal a la información y al conocimiento } \\
\text { (UNESCO, 2003) Citado en Castañeda y otros (2007). }\end{array}$ \\
\hline $\begin{array}{l}\text { Sostenibilidad Ciudadana } \\
\text { y Democracia }\end{array}$ & $\begin{array}{l}\text { Fomentando valores, participación ciudadana, deberes } \\
\text { ciudadanos y un dialogo democrático. }\end{array}$ \\
\hline Calidad & $\begin{array}{l}\text { Calidad total buscando el mejoramiento continuo, } \\
\text { formación, desarrollo y productividad. }\end{array}$ \\
\hline $\begin{array}{l}\text { Compromiso con la } \\
\text { Comunidad }\end{array}$ & $\begin{array}{l}\text { La universidad toma recursos que la comunidad da a su } \\
\text { disposición, por lo tanto es deber de ella retribuirle. }\end{array}$ \\
\hline Marketing Responsable & $\begin{array}{l}\text { Que enfatiza el hecho de que una idea sea aceptada en la } \\
\text { sociedad. }\end{array}$ \\
\hline Imagen Institucional & $\begin{array}{l}\text { La misión de la universidad refleja un conjunto de } \\
\text { propiedades que determinan su imagen ante la sociedad. }\end{array}$ \\
\hline
\end{tabular}

Fuente: elaboración propia a partir de Castañeda y otros (2007) 
La universidad debe estar en armonía con la sociedad, buscando una mejor manera de actuar y poderse proyectar generando un buen impacto, es por eso que también la RSU evalúa los impactos de una universidad, estos pueden ser organizacionales, educacional, medioambiental y cognitivo con el propósito de promover un desarrollo sostenible. Castañeda y otros (2007) y De la Red Vega (2009), Aclarando conceptos Vivas (2012) diferencia actos de impactos, donde los actos son los que realiza una persona, por consiguiente puede controlarlos en y los impactos que se generan colectivamente. Aclarado esto al referirse a impactos Vallaeys (2013) los define de la siguiente manera.

- Impactos organizacionales: comprenden aspectos laborales, ambientales, de hábitos de vida cotidiana en el campus.

- Impactos educativos: procesos de enseñanza, aprendizaje y la creación de un perfil de egresado que se está formando.

- Impactos cognitivos: se relacionan con las orientaciones epistemológicas y deontológicas, los enfoque teóricos y las líneas de investigación, los procesos de producción y difusión del saber, que derivan en el modo de gestión del conocimiento.

- Impactos sociales: vínculos que tiene la Universidad con la comunidad como promotora del desarrollo humano sostenible.

Aristimuño y Rodríguez (2014) agregan a estos impactos la parte ambiental, que está determinada por el "conjunto de acciones que establece la gerencia para la incorporación de los aspectos medioambientales y sus impactos”. Estos impactos en caso de que sean negativos se les hace una mejora continua.

En busca de ser mejor personas aparece la empatía entendida como "un proceso de regulación cognitiva y emocional que facilita la inhibición de comportamientos disóciales, y reconocimiento de las emociones en los otros como factor esencial para establecer procesos empáticos y las habilidades prosociales, morales y éticas", es decir que el estudiante posea la habilidad de vincularse empáticamente con los demás (Arango, Clavijo, Puerta y Sánchez, 2014).

\subsection{La Misión universitaria y su relación con la RSU}

Es importante que si se habla de RSU, se mencione directamente la misión de la universidad ya que dentro de las funciones de una universidad está la proyectar su misión por medio de "la enseñanza, investigación y servicio" (Declaración de Taillores, 2005); por su parte en Colombia, la Asociación Colombina de Universidades 
ASCUN (2011) hace una reflexión de que la visión y la misión de una universidad son un marco de referencia para que las universidades realicen su autoevaluación, buscando calidad y excelencia institucional.

Ganga y Navarrete (2012) que destacan que "la responsabilidad que las instituciones de educación superior tienen frente a la sociedad proviene directamente de su misión social, en la cual se establecen las funciones principales de una universidad, las que abarcan ámbitos como la docencia, la investigación, la vinculación con el medio y la gestión universitaria”. En países como Argentina, Venezuela, México, Costa Rica y Colombia los valores son los principios fundamentales que guían las misiones de algunas de sus universidades (Herrera y otros, 2009).

Dentro de este marco se puede considerar la RSU (Arango y otros, 2014) como el análisis y reflexión que realiza la institución de su misión en cuanto a las contribuciones con la sociedad y a la formación que les proporciona a sus estudiantes lo que apoya Bustos e Inciarte (2012) concluyen que "la existencia de la universidad se justifica en tanto y en cuanto que, la institución esté en sintonía y al servicio de la comunidad en general y eso sólo se logra a través de una visión y misión compartida social y éticamente deseable, viable y posible, a lo interno de ella y más allá de los espacios universitarios a través del cumplimiento permanente, activo, creativo, pertinente, efectivo y eficaz de las tres funciones básicas universitarias y la gestión administrativa institucional”.

\subsection{Universidades latinoamericanas y su RSU}

En una investigación realizada por García y Alvarado (2012), muestran que en el caso particular en la universidad de Zulia (Venezuela) cuyo propósito era analizar la RSU de dicha universidad, estos autores concluyen que la universidad debe ayudar a la integración social, donde el pensum de estudio contenga asignaturas de responsabilidad social para ayuda de las comunidades y del mundo. Arango y otros (2014) en uno de sus estudios realizados sobre empatía, valores y comportamientos socialmente responsables de los estudiantes de la universidad, obtienen como resultado que los estudiantes tienen autocuidado por el medio ambiente y respetan los espacios que comparten, pero que esto se alimenta más con formación académica fundamentada en valores, ética, también en actividades de voluntariado, ayuda social, cuidado ecológico y responsabilidad cívica.

En una investigación realizada por Recalde, Cantero y Jara (2012) donde se cuestionaba si la universidad objeto de estudio (Universidad Nacional de Caaguazú - Brasil) cumplía una función integradora de RSU se obtuvo como resultado que por 
medio de la extensión, la universidad propone involucrarse en la realidad nacional y departamental mediante actividades y proyectos coherentes como en este caso que buscaba la prevención y promoción de la salud en los niños.

Hoy en día es necesario crear esa colectividad con la sociedad ya que la mayoría de universidades como lo menciona Herrera y otros (2009) poseen infraestructuras que les permiten crear investigación científica y desarrollo tecnológico, por ejemplo están las universidades como UNC con museo astronómico y BUAP, UNAM con centro de prevención de desastres, otras universidades incursionan en avances tecnológicos, dependiendo de sus disciplinas y áreas de conocimiento, lo que permite a las universidades a estar alertas en la trasformación del entorno.

Estas son algunas de las experiencias de universidades latinoamericanas, en cuanto a su RSU, por otro lado, las que son consideras como las mejores universidades de América latina, (por medio del estudio previo realizado donde se miraba su extensión universitaria), mostraron que es de gran importancia conocerlo y aprender a entenderlo ya que este es un pilar que guía a la universidad para que busque una responsabilidad social, vemos adicional a esto que países como en Chile potencian sus esfuerzos a la comprensión de la responsabilidad social universitaria, donde se crean redes, se realizan congresos, jornadas y conferencia que reúnen e integran a otras universidades del entorno, en miras a compartir experiencias que mejoren el actuar de cada universidad, es de resaltar que en este país se crea un proyecto importante que es el de Universidad construye país de Jiménez, De Ferrari, Delpiano y Ardiles. C. (2004). Cada uno de estos países por medio de sus universidades genera grandes conocimientos y publicaciones de gran impacto en torno a la RSU.

\subsection{Colombia frente a la RSU}

Por otra parte tiene también participación en el Observatorio de Responsabilidad Social Universitaria (ORSU) que se hace cargo de desafíos encaminados a la responsabilidad ciudadana, por medio de la formación de profesionales que sean capaces de hacer gestión de ciudadanía, valores y al mismo tiempo por medio de métodos innovadores que favorezcan a la sociedad, adicional a esto se encuentra el Observatorio de Responsabilidad Social de las Universidades a Distancia OIRSUD que vincula universidades a distancia para que unan esfuerzos en la realización de foros de responsabilidad social, RSU y el cambio de paradigmas a una educación que impulsa a estas instituciones a sacarle provecho de las TIC para soportar sus procesos; dentro de estas instituciones Colombia cuenta con la participación de la UNAD (Hernández, 2011). 
Hernández y Saldarriaga (2009), en un caso particular en la universidad de Antioquia, su escuela de ingeniera IEA propone un concepto de RSU encaminado hacia su universidad el cual es llevar a la práctica el conjunto principios y valores que hacen a la EIA corresponsable en la construcción de una mejor sociedad, sociedad en la cual la institución identifica los impactos de su labor y propicia efectos positivos con criterios de sostenibilidad.

Otra de las experiencias que se llevó a cabo en la Universidad de Antioquia fue un encuentro denominado "Catedra Abierta de Responsabilidad Social Universitaria" donde reunió a varios expertos en tema de RSU como medio de autoevaluación, con el fin de consolidar experiencias en lo relacionado con el campus responsable, formación profesional y ciudadana, y la participación social, también sirvió para contribuir a la comprensión de componentes y procesos que implican su desarrollo; se resalta que espacios como estos brindan profundizar y aclarar el concepto de RSU (Vivas, 2012). Esto muestra que este tipo de eventos no son pasados por alto y que en caso de esta universidad se les han dado gran importancia y reconocimiento.

Por otra parte se aprecia que las experiencias de las universidades en Colombia e cuanto RSU son diversas, por ejemplo en la Universidad de Antioquia donde se llevan a cabo eventos de gran importancia como los que ya se mencionaron anteriormente, y adicional a esto en el año 2013 se lleva a cabo un encuentro de RSU de la Red Universitaria de Extensión y Proyección de Antioquia, cuyo objetivo era el de "generar un espacio para que universidades presenten sus modelos, políticas e indicadores de Responsabilidad Social Universitaria, los cuales puedan constituirse en referentes para otras Instituciones de Educación Superior". Está conto con la participación de las universidades que hacen parte de esa red (Corporación Universitaria Lasallista, Escuela de Ingeniería de Antioquia, Instituto Tecnológico Metropolitano, Politécnico Colombiano Jaime Isaza Cadavid, Universidad CES, Universidad Cooperativa de Colombia, Universidad de Antioquia, Universidad de Medellín, Universidad de San Buenaventura, Universidad EAFIT, Universidad Nacional de Colombia y la Universidad Pontificia Bolivariana.

La Universidad ICESI (s.f) maneja su RSU por medio de políticas como la de una gestión institucional responsable, que también encierra al medio ambiente, una política de formación ciudadana en el proyecto educativo de ICESI, otra de investigación de participación social en la que se encuentra su proyecto social universitario. Al igual que la Universidad Javeriana la RSU es tratada por medio una política de responsabilidad social cuyo objetivo principal es el de crear desarrollo positivo que oriente su actuar a la transformación constructiva de la sociedad en que vive. Por su 
lado, la Universidad EAFIT (2009) entre sus principales objetivos de responsabilidad social están el de contribuir con la formación personal ético, líder y proactivo.

En un foro Responsabilidad Social en la Educación Superior en cuanto a la experiencia de la Universidad del Valle, muestra como esta universidad ha tenido logros en cuanto a la RSU como desarrollo de acciones de relación con toda la sociedad, apoyo a poblaciones vulnerables e indígenas, evita la deserción estudiantil, por su parte es una universidad que integra de la mejor manera la investigación, la docencia y la extensión (Ramos, 2009).

Se concluye que estas universidades manejan su responsabilidad de diversas maneras pero todas apuntan a mejorar la sociedad, por medio de proyectos sostenibles, esto a su vez, ayuda a que por medio de estas IES se empiece a transformar el país, donde prime lo justo. Cada universidad busca también que sus estudiantes sean formados de tal manera que se conviertan en excelentes profesionales éticos que respondan ante la sociedad y sean parte de la solución de sus necesidades.

Es importante entonces reconocer los grandes esfuerzos y como lo expresa Hernández y Saldarriaga (2009) se mencionan proyectos de RSU premiados por el Banco Interamericano de Desarrollo (BID).

a. Pontificia Universidad Javeriana Cali (coordinadora). Objetivo: la generación de una Red Universitaria en RSU en la ciudad de Santiago de Cali.

b. Proyecto RSU Universidad de Antioquia / Banco Interamericano de Desarrollo. Objetivo: fortalecer la capacidad de la Universidad para incorporar en el desarrollo de su misión, la práctica sistemática y sostenida de la RSU.

c. La RSU en la Universidad Cooperativa de Colombia. Objetivos: la construcción de un modelo educativo para el desarrollo de la responsabilidad ética y social universitaria e implementar el currículo del área institucional con un enfoque de responsabilidad social universitaria ética y desarrollo.

d. Modelo Estructural y Dinámico de Responsabilidad Social, Ética y Desarrollo, en la Universidad de Ibagué -Coruniversitaria-. Objetivo: crear un modelo estructural y dinámico de responsabilidad social para el desarrollo interno (intrínseco) y externo (extrínseco) de la universidad de Ibagué-Coruniversitaria, y unos indicadores para medir el impacto de las actividades. 
e. Construcción del êthos universitario de la Universidad Industrial de Santander como principio de autorregulación para la definición y apropiación de su protocolo ético.

Se puede demonstrar que Colombia participa en la búsqueda de su responsabilidad social universitaria de manera importante, ya que al recibir estos reconocimientos que se les ha otorgado a estas universidades, de una u otra manera se incentiva al resto de las universidades del país a seguir su ejemplo.

Por otro lado, en un análisis similar a que se le realizo a las universidades Latinoamericanas, donde se observó en las páginas web de la universidad su extensión o proyección social se pudo evidenciar que a través de decretos, acuerdos y resoluciones cada universidad estipula ciertos principios o lineamientos que le sirven para interactuar con la comunidad de la cual hacen parte, dependiendo el sector en el que se encuentre dicha universidad ayuda a dar solución a sus problemas de manera oportuna con principios como excelencia académica, pertinencia, integralidad, comunicación, cooperación, solidaridad, formación, ética, transparencia, pertinencia social entre otros, son los que guían a muchas de estas universidades siguen para mejorar mutuamente con la sociedad, ya que bajo estos principios guían sus esfuerzos.

Se evidenció que por medio de la extensión, la mayoría de las universidades buscan cumplir con su misión institucional, que como se dijo anteriormente es de gran importancia, puesto que esta es su razón de ser y debe estar bien planteada y guiada hacia el desarrollo y bienestar de la sociedad, por consiguiente la extensión es parte fundamental de la misión de las universidades colombianas que se ven reflejadas por medio de asesorías, capacitaciones, ciencia, tecnología, arte y cultura, es decir por medio de diferentes áreas académicas e investigativas, la universidad genera conocimiento dependiendo de lo que la sociedad necesite.

Con esta interacción que hace la universidad con la comunidad se genera un compromiso mutuo, y cabe resaltar que esta interacción que realiza la universidad la hace también con el estado, el sector público, privado, ONG, sociedad civil en general, donde a través de proyectos que en primera instancia son planteados por la misma sociedad, la universidad de una u otra manera le ayuda a dar solución a estos problemas, buscando siempre el desarrollo social, la sostenibilidad y transformación social. La extensión universitaria busca también el desarrollo económico, cultural, ambiental, político y tecnológico del país. Vemos que cada universidad por medio de estas propuestas o escritos de extensión refleja ya en primera instancia ese compromiso por la comunidad de la cual hace parte, también se evidencia que no es tan solo como 
este planteada su extensión sino que esta a su vez debe estar bien respaldada por la docencia y la investigación, para que se dé un acercamiento a la verdadera responsabilidad social universitaria.

\section{Conclusiones}

En un mundo de injusticia como el que se está dando, es importante que las personas empiecen a generar conciencia de lo que sus actos generan, en especial las comunidades universitarias, ya que por medio de su formación ética tienen la capacidad de reflexionar sobre esas problemáticas y poseen los conocimientos para ayudar a dar solución a esas injusticias.

La RSU, posee innumerables fuentes de información que se esparcen a lo largo del mundo, por medio de pequeñas investigaciones esta, la información se va filtrando lo que conlleva a hacer mejores análisis y sobretodo comprender lo que esta abarca, no todo está dicho en cuanto a la RSU, pero contribuciones de este tipo de ayudan a que se generen debates concientización.

La manera como se mide la RSU, es a través de sus impactos por lo que la universidad no está exenta de generar impactos negativos, pero se evidencia que por medio de la responsabilidad social universitaria se pueden contrarrestan, creando vínculos con la comunidad por medio de los procesos claves que muchos de estos autores concuerdan, es decir docencia, gestión, investigación y extensión. También es importante que la universidad conozca sus stakeholders, ya que por medio de este simple reconocimiento, se empiezan a generar los lazos de apoyo e integración con los mismos para que todos apunten al mismo fin, es decir a una sociedad sustentable; por otra parte, la interacción entre universidades ayuda a que por medio de experiencias compartidas se refuercen debilidades y se potencien las fortalezas que ya se poseen.

Para un estudiante es importante conocer la misión de la IES a la cual pertenece, ya que por medio de este simple reconocimiento, este empieza a generar conciencia y compromiso moral de que como futuro profesional debe proyectar ese perfil que la universidad le ha otorgado.

En Colombia se aprecia que las universidades están cada vez más interesadas en el desarrollo de la RSU, sus iniciativas son valiosas y sus proyectos notables, pues la mayoría de estos proyectos se crean para y con la sociedad, donde se ayudan a los más vulnerables y se apoya a los que quieren salir adelante y progresar. Las universidades muestran que se están formando personas con principios éticos y con 
valores, también personas líderes que son capaces de afrontar desafíos y nuevos retos que se van generando con el transcurso del tiempo. Como lo manifiesta De la red Vega (2009) al decir "la universidad, como organización, es un escenario privilegiado para la potenciación de recursos humanos y sociales y para la promoción de profesionales socialmente responsables en un mundo con nuevas necesidades". Se puede precisar que son las IES las que abanderan los procesos de modernización de la sociedad de manera ética y responsable, por lo tanto se hace necesario estudiar las experiencias en RSU incluso para ser llevadas a cabo a nivel empresarial.

\section{Referencias}

Aguirre, R., de Pelekais, C. y Paz, A. (2012). Responsabilidad social: compromiso u obligación universitaria. Telos, 14(1), 11-20.

Asociación Colombiana de Universidades - ASCUN, (2011). Responsabilidad Social Universitaria - RSU. Pensamiento universitario no 21. Colombia: Asociación Colombiana de Universidades.

Arango, O., Clavijo, S., Puerta, I. y Sánchez, J. (2014). Formación académica, valores, empatía y comportamientos socialmente responsables en estudiantes universitarios. Revista de la Educación Superior, 43 (169) 89-105.

Aristimuño, M. y Rodríguez, C. (2014). Responsabilidad social universitaria. Su gestión desde la perspectiva de directivos y docentes. Estudio de caso: una pequeña universidad latinoamericana. Interciencia, 39(6), 375-382.

Bernal, H. y Rivera, V. (2011). Responsabilidad social universitaria: Aportes para el análisis de un concepto. ASCUN. Pensamiento universitario, (21), 7-17.

Bustos, C. e Inciarte, A. (2012). Dimensión comunitaria de la responsabilidad social universitaria. Opción, 28(68), 367-379.

Casilla, D. y Camacho, H. (2012). Evaluación de la Responsabilidad Social Universitaria. Opción, 28(69), 452-465.

Castañeda, G., Ruiz, M., Viloria, O., Castañeda, R. y Quevedo. (2007). El rol de las universidades en el contexto de la responsabilidad social empresarial. Negotium, 3(8), 100-132.

Comunidad europea de Naciones. (2001). Fomentar un marco europeo para la responsabilidad social de las empresas: Libro Verde. Bruselas: Comisión de las Comunidades. 
Consejo Nacional de Acreditación - CNA (2014). Cifras del sistema nacional de acreditación a 30 de junio de 2014 - 1. Bogotá: Consejo Nacional de Acreditación, 1-69.

Castro, M. (2005). La Responsabilidad Social de las Empresas, o un nuevo concepto de empresa. CIRIEC-España. Revista de Economía Pública, Social y Cooperativa, (53), 29-51.

De la Red Vega, N. (2009). Necesidades emergentes y responsabilidad social universitaria. Alternativas. Cuadernos de Trabajo Social, (16), 65-76.

Declaración de Talloires (2005). Sobre las responsabilidades cívicas sociales y las funciones cívicas de la educación superior. Massachusetts: Tufts University, $1-3$.

Drucker, P. (1995). Drucker. Su visión sobre: la administración. La organización basada en la información. La economía. La sociedad. Bogotá: Editorial Norma.

Drucker, P. (2006). Drucker para todos los días. 336 días de reflexiones claves para acertar en su negocio. Bogotá: Editorial Norma.

Ganga, F. y Navarrete, E. (2012). Universidades privadas y su responsabilidad social en Chile: Un estudio exploratorio. Opción, 28(68), 243-256.

García, C. y Alvarado, Y. (2012). Responsabilidad social universitaria como plataforma para el desarrollo de la comunidad. Multiciencias, 12, 59-63.

Gasca, E. y Olvera, J. (2011). Construir ciudadanía desde las universidades, responsabilidad social universitaria y desafíos ante el siglo XXI. Convergencia. Revista de Ciencias Sociales, 18(56), 37-58.

González, E. (2007). La teoría de los stakeholders. Un puente para el desarrollo practico de la ética empresarial y de la responsabilidad social corporativa. Veritas revista de Filosofía y Teología, 2(17), 205-224.

González, N., Pirela, A. y Zerpa, M. (2012). La formación docente como investigador. Una Responsabilidad Social Universitaria. Opción, 28(69), 466-479.

Hernández, Á (2011). Observatorio de Responsabilidad Social de las Universidades a Distancia (OIRSUD). Boletín Iesalc Informa de educación superior, (214). Recuperado de: http://iesalc.unesco.org.ve/index.php?option=com_ content $\&$ view $=$ article $\&$ id $=2593 \% 3$ Aobservatorio-de-responsabilidad-socialde-las-universidades-a-distancia-oirsud\&catid=126\%3 Anoticias-paginanueva\&Itemid=712\&lang=es. 
Hernández, D. y Saldarriaga, A. (2009). Gestión de la responsabilidad social universitaria. Caso: Escuela de Ingeniería de Antioquia (EIA). Dyna, 76(159), 237-248.

Herrera, M., Didriksson, T. y Sánchez, C. (2009). La responsabilidad social en las macrouniversidades públicas de América Latina y el Caribe. Universidades, 59(41) 11-26.

Jiménez. M., De Ferrari. J., Delpiano, C y Ardiles. C. (2004). Universidad Construye País, "Observando la Responsabilidad social universitaria”. México: Universidad Internacional - UNINTER.

Martinez, H (2011). Responsabilidad social y ética empresarial. Bogotá: Ecoe Ediciones.

Martínez, P. y Hernández, A. (2013). Responsabilidad social universitaria: un desafío de la universidad pública mexicana. Contribuciones desde Coatepec, (24), 85-103.

Organización para la Cooperación y el Desarrollo Económicos - OECD, (2014). The Organisation for Economic Co-operation and Development. Recuperado de: http://www.oecd.org/about/.

Ramos. I. (2009). Foro Responsabilidad Social en la Educación Superior: "La Experiencia de la Universidad del Valle”. Foro. Universidad del valle: Bogotá, agosto 13 y 14 de 2009, 1-6.

Recalde, H., Cantero, W. y Jara, J. (2012). Importancia de la responsabilidad social universitaria como desarrollo social de la ciudad de Coronel Oviedo. Revista Gestão Universitária na América Latina - GUAL, 5(4), 266-280.

Social Accountability International - SAI, (2015). Advancing the human rights of workers around the world. Recuperado de: http://www.sa-intl.org/.

Organización de las Naciones Unidas para la Educación, la Ciencia y la Cultura UNESCO, (1998). Conferencia Mundial sobre Educación Superior, Declaración mundial sobre la educación superior en el siglo XXI: visión y acción. Recuperado de: http://www.unesco.org/education/educprog/wche/ declaration_spa.htm

Universidad EAFIT. (2009). EAFIT, En comunicación directa con el mundo. Recuperado de: http://www.eafit.edu.co/EafitCn/Noticias/EAFIT+en+ comunicacion+directa+con+la+region.htm. 
Universidad ICESI. (s.f). Política de responsabilidad Social Universitaria ICESI. Colombia: ICESI, 1-15.

Vallaeys, F. (2013). La responsabilidad social universitaria: ¿cómo entenderla para quererla y practicarla?. Perú: Pontificia Universidad Católica del Perú, 1-17.

Vallaeys, F. (2014). La responsabilidad social universitaria: un nuevo modelo universitario contra la mercantilización. Revista Iberoamericana de Educación Superior (RIES). 105-117.

Vallejo, R. y Govea, M. (2011). Responsabilidad social e investigación: retos de la universidad del siglo XXI. Telos, 13(2), 216-236.

Vera, L., Gómez, M., Acosta, Y. y Perozo, L. (2012). La docencia en el marco de la responsabilidad social universitaria. Opción, 28(68) 257-272.

Vivas, M. (2012). Responsabilidad social universitaria, autoevaluación del sistema universitario de extensión. Memorias. Octava Cátedra Abierta de Extensión 2012. Colombia: Universidad de Antioquia. 
